\title{
Verifying C11-Style Weak Memory Libraries
}

\author{
Sadegh Dalvandi and Brijesh Dongol * \\ University of Surrey, Guildford, UK \\ $\{\mathrm{m}$. dalvandi, b. dongol\}@surrey.ac.uk
}

\begin{abstract}
Deductive verification of concurrent programs under weak memory has thus far been limited to simple programs over a monolithic state space. For scalabiility, we also require modular techniques with verifiable library abstractions. This paper addresses this challenge in the context of RC11 RAR, a subset of the C11 memory model that admits relaxed and release-acquire accesses, but disallows, so-called, load-buffering cycles. We develop a simple framework for specifying abstract objects that precisely characterises the observability guarantees of abstract method calls. We show how this framework can be integrated with an operational semantics that enables verification of client programs that execute abstract method calls from a library it uses. Finally, we show how implementations of such abstractions in RC11 RAR can be verified by developing a (contextual) refinement framework for abstract objects. Our framework, including the operational semantics, verification technique for client-library programs, and simulation between abstract libraries and their implementations, has been mechanised in Isabelle/HOL.
\end{abstract}

\section{Introduction}

An effective technique for reasoning about weak memory models is to consider the observations that a thread can make of the writes within a system. For example, for certain subsets of C11 (the 2011 C standard), reasoning about per-thread observations has led to operational characterisations of the memory model, high-level predicates for reasoning about per-thread observations, and deductive verification techniques applied to standard litmus tests and synchronisation algorithms [5]. Current verification techniques are however, focussed on (closed) programs, and hence do not provide any mechanism for (de)composing clients and libraries. This problem requires special consideration under weak memory since the execution of a library method induces synchronisation. That is, a thread's observations of a system (including of client variables) can change when executing library methods.

This paper addresses several questions surrounding client-library composition in a weak memory context.

(1) How can a client use a weak memory library, i.e., what abstract guarantees can a library provide a client program? Prior works [13, 3] describe techniques for specifying the behaviour

${ }^{*}$ This work is supported by EPSRC Grant EP/R032556/1. 


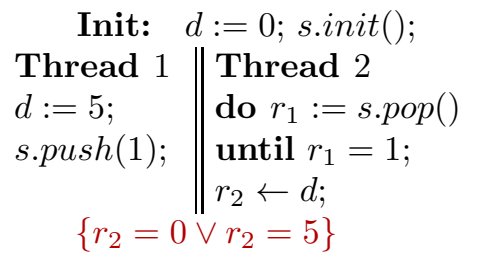

Figure 1: Unsynchronised message passing

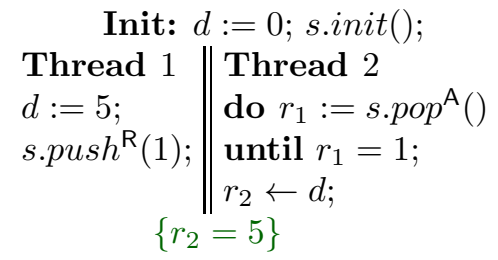

Figure 2: Publication via a synchronising stack

of abstract objects, which are in turn related to their implementations using causal relaxations of linearizability. However, these works do not provide a mechanism for reasoning about the behaviour of client programs that use abstract libraries. In this paper, we address this gap by presenting a modular operational semantics that combines weak memory states of clients and libraries.

(2) What does it mean to implement an abstract library? To ensure that behaviours of client programs using an abstract library are preserved, we require contextual refinement between a library implementation and its abstract specification. This guarantees that no new client behaviours are introduced when a client uses a (concrete) library implementation in place of its (abstract) library specification. Under sequential consistency (SC), it is well known that linearizable libraries guarantee (contextual) refinement [12, 16, 15]. However, under weak memory, a generic notion of linearizability is difficult to pin down [13, 8]. We therefore present a direct technique for establishing contextual refinement under weak memory. A key innovation is the development of context-sensitive simulation rules that ensures that each client thread that uses the implementation observes a subset of the values seen by the abstraction.

(3) Can the same abstract library specify multiple implementations? A key benefit of refinement is the ability to use the same abstract specification for multiple implementations, e.g., to fine-tune clients for different concurrent workload scenarios. To demonstrate applicability of our framework, we provide a proof-of-concept example for an abstract lock and show that the same lock specification can be implemented by a sequence lock and ticket lock. The theory itself is generic and can be applied to concurrent objects in general.

(4) How can we support verification? Can the verification techniques be mechanised? Assuming the existence of an operational semantics for the underlying memory model, we aim for deductive verification of both client-library composition and contextual refinement. We show that this can be supported by prototyping the full verification stack in the Isabelle/HOL theorem prover 1 .

\section{Message passing via library objects}

In this section, we illustrate the basic principles of client-object synchronisation in weak memory.

Client-object message passing. Under SC all threads have a single common view of the shared state. When a new write is executed, the "views" of all threads are updated so that they are guaranteed to only see this new write. In contrast, each thread in a C11 program has its own view of each variable. Views may not be updated when a write occurs, allowing threads to read stale writes. To enforce view updates, additional synchronisation (e.g., release-acquire) must be introduced [4, 18, 23].

Now consider a generalisation of this idea to (client) programs that use library objects. The essence of the problem is illustrated by the message-passing programs in Figures 1 and 2 , Under

\footnotetext{
${ }^{1}$ Our Isabelle theories may be accessed as ancillary material in the ArXiV submission.
} 


$$
\begin{aligned}
& \text { Init: } \quad d:=0 ; \text { s.init }() \text {; } \\
& \left\{[d=0]_{1} \wedge[d=0]_{2} \wedge\left[\text { s.pop }_{\text {emp }}\right]_{1} \wedge\left[\text { s.pop }_{\text {emp }}\right]_{2}\right\} \\
& \begin{array}{l||l}
\text { Thread } 1 & \text { Thread } 2 \\
\left\{\neg\langle\text { s.pop }\rangle_{2} \wedge[d=0]_{1}\right\} & \left.\left\{\langle\text { s.pop }\rangle_{1}\right\rangle[d=5]_{2}\right\} \\
1: d:=5 ; & 3: \text { do } r_{1}:=\text { s.pop } \\
& \text { A }() \\
\left\{\neg\langle\text { s.pop }\rangle_{2} \wedge[d=5]_{1}\right\} & \text { until } r_{1}=1 ; \\
2: \text { s.push }^{\mathrm{R}}(1) ; & \left\{[d=5]_{2}\right\} \\
\text { \{true }\} & 4: r_{2} \leftarrow d ; \\
& \left\{r_{2}=5\right\} \\
& \left\{r_{2}=5\right\}
\end{array}
\end{aligned}
$$

Figure 3: A proof outline for message passing

$\mathrm{SC}$, when the program in Figure 1 terminates, the value of $r_{2}$ is guaranteed to be 5 . However, this is not necessarily true in a weak memory setting. Even if pop operation in thread 2 returns 1, it may be possible for thread 2 to observe stale value 0 for $d$. Therefore the program only guarantees the weaker postcondition $r_{2}=0 \vee r_{2}=5$.

To address this problem, the library operations in Figure 2 are annotated with release-acquire annotations. In particular, the client assumes the availability of a "releasing push" $\left(\right.$ push $\left.^{\mathrm{R}}(1)\right)$, which is to be used for message passing. Thread 2 pops from $s$ using an "acquiring pop" $\left(p_{p} p^{\mathrm{A}}()\right)$. If this pop returns 1 , the stack operations induce a happens-before synchronisation in the client, which in turn means that it is now impossible for thread 2 to read the stale initial write for $d$.

Verification strategy. Our aim is to enable deductive verification of such programs by leveraging recently developed operational semantics, assertion language and Owicki-Gries style proof strategy for RC11 RAR [5]. We show that these existing concepts generalise naturally to client-object, and in a manner that enables modular proofs.

The assertion language of 5 enables reasoning about a thread's views, e.g., in Figure 3 , after initialisation, thread $t \in\{1,2\}$ has definite value 0 for $d$ (denoted $[d=0]_{t}$ ).

In this paper, we extend such assertions to capture thread views over library objects. E.g., after initialisation, the only value a pop by thread $t$ can return is Empty, and this is captured by the assertion [s.popemp $]_{t}$. The precondition of $d:=5$ states that thread 2 cannot pop value 1 from $s$ (as captured by the assertion $\neg\left\langle s . p o p_{1}\right\rangle_{2}$ ). The precondition of the until loop in thread 2 contains a conditional observation assertion (i.e., $\langle$ s.pop 1$\rangle[d=5]_{2}$ ), which states that if thread 2 pops value 1 from $s$ then it will subsequently be in a state where it will definitely read 5 for $d$.

A key benefit of the logic in [5] is that it enables use of standard Owicki-Gries reasoning and straightforward mechanisation [6]. As we shall see (Section [5.3), we maintain these benefits in the context of client-object programs.

Contextual refinement. Contextual refinement relates a client using an abstract object with a client that uses a concurrent implementation of the object. More precisely, we say that a concrete object $C O$ is a contextual refinement of an abstract object $A O$ iff for any client $C$, every behaviour of $C$ when it uses $C O$ is a possible behaviour of $C$ when it uses $A O$. Thus, there is no observable difference to any client when it uses $C O$ in place of $A O$.

In a weak memory setting, to enable a client to use an object, one must specify how synchronisation between object method calls affects the client state. To implement such a specification, we must describe how the abstract synchronisation guarantees are represented in the implementa- 
tion. Prior works have appealed to extensions of notions such as linearizability to ensure contextual guarantees [13, 14, 26. In this paper, we aim for a more direct approach and consider contextual refinement directly.

\section{Generalised operational semantics}

We now present a simple program syntax that allows one to write open programs that can be filled by an abstract method or concrete implementation of a method.

\subsection{Program Syntax}

We start by defining a syntax of concurrent programs, starting with the structure of sequential programs (single threads). A thread may use global shared variables (from GVar) and local registers (from $L V a r$ ). We let $\operatorname{Var}=G \operatorname{Var} \cup L \operatorname{Var}$ and assume $G \operatorname{Var} \cap L V a r=\emptyset$. For client-library programs, we partition $G \operatorname{Var}$ into $G \operatorname{Var}_{C}$ (the global client variables) and $G \operatorname{Var}_{L}$ (the global library variables) and similarly $L V a r$ into $L \operatorname{Var}_{C}$ and $L V a r_{L}$. In an implementation, global variables can be accessed in three different synchronisation modes: acquire ( $\mathrm{A}$, for reads), release ( $\mathrm{R}$, for writes) and relaxed (no annotation). The annotation RA is employed for update operations, which reads and writes to a shared variable in a single atomic step. We let $O b j$ and Meth be the set of all objects and method calls, respectively.

We assume that $\ominus$ is a unary operator (e.g., $\neg$ ), $\oplus$ is a binary operator (e.g., $\wedge,+,=$ ) and $n$ is a value (of type $\mathrm{Val}$ ). Expressions must only involve local variables. The syntax of sequential programs, Com, is given by the following grammar with $r \in L V a r, x \in G \operatorname{Var}, o \in O b j, m \in$ Meth, $u, v \in$ Val:

$$
\begin{aligned}
& \operatorname{Exp}_{L}::=\operatorname{Val}|L \operatorname{Var}| \ominus \operatorname{Exp}_{L} \mid \operatorname{Exp}_{L} \oplus \operatorname{Exp}_{L} \\
& \operatorname{CExp}_{L}::=\bullet \mid \operatorname{Exp}_{L} \\
& \text { • ::= Val } \mid \text { o.m }([u]) \mid \text { Com, where Com contains no holes } \\
& A C o m::=\bullet|\perp| r \leftarrow \mathbf{C A S}(x, u, v)^{\mathrm{RA}}\left|r \leftarrow \mathbf{F A I}(x)^{\mathrm{RA}}\right| r:=\operatorname{CExp}_{L}\left|x:={ }^{[\mathrm{R}]} \operatorname{Exp}_{L}\right| r \leftarrow{ }^{[\mathrm{A}]} x \\
& C o m::=A C o m|C o m ; C o m| \text { if } B \text { then } C o m \text { else Com } \mid \text { while } B \text { do Com }
\end{aligned}
$$

where we assume $B$ to be an expression of type $C \operatorname{Exp}_{L}$ that evaluates to a boolean. We allow programs with holes, denoted $\bullet$, which may be filled by an abstract or concrete method call. During a program's execution, the hole may also be filled by the null value $\perp \notin V a l$, or the return value of the method call. The notation $[X]$ denotes that the annotation $X$ is optional, where $X \in\{A, R\}$, enabling one to distinguish relaxed, acquiring and releasing accesses. Within a method call, the argument $u$ is optional. Later, we will also use do-until loops, which is straightforward to define in terms of the syntax above.

\subsection{Program Semantics}

For simplicity, we assume concurrency at the top level only. We let Tid to be the set of all thread identifiers and use a function Prog: Tid $\rightarrow$ Com to model a program comprising multiple threads. In examples, we typically write concurrent programs as $C_{1}\|\ldots\| C_{n}$, where $C_{i} \in C o m$. We further assume some initialisation of variables. The structure of our programs thus is Init; $\left(C_{1}\|\ldots\| C_{n}\right)$. 


$$
\begin{aligned}
& \frac{r \in L \operatorname{Var} \quad v=\llbracket E \rrbracket_{l s}}{(r:=E, l s) \stackrel{\epsilon}{\rightarrow}(\perp, l s[r:=v])} \quad \frac{x \in G \operatorname{Var} \quad a=w r^{[\mathrm{R}]}(x, \llbracket E \rrbracket l s)}{\left(x:={ }^{[\mathrm{R}]} E, l s\right) \stackrel{a}{\rightarrow}(\perp, l s)} \\
& \frac{a=r d^{[\mathrm{A}]}(x, v) \quad v \in V a l}{\left(r \leftarrow{ }^{[\mathrm{A}]} x, l s\right) \stackrel{a}{\rightarrow}(\perp, l s[r:=v])} \\
& \frac{\left(C_{1}, l s\right) \stackrel{a}{\rightarrow}\left(C_{1}^{\prime}, l s^{\prime}\right)}{\left(C_{1} ; C_{2}, l s\right) \stackrel{a}{\rightarrow}\left(C_{1}^{\prime} ; C_{2}, l s^{\prime}\right)} \quad \frac{v \in V a l \cup\{\perp\}}{\left(v ; C_{2}, l s\right) \stackrel{\epsilon}{\rightarrow}\left(C_{2}, l s\right)} \\
& \frac{\llbracket B \rrbracket_{l s}}{(I F, l s) \stackrel{\epsilon}{\rightarrow}\left(C_{1}, l s\right)} \quad \frac{\neg \llbracket B \rrbracket l s}{(I F, l s) \stackrel{\epsilon}{\rightarrow}\left(C_{2}, l s\right)} \\
& \frac{\llbracket B \rrbracket_{l s}}{(\text { WHILE, ls }) \stackrel{\epsilon}{\rightarrow}(C ; W H I L E, l s)} \quad \frac{\neg \llbracket B \rrbracket_{l s}}{(\text { WHILE,ls } \stackrel{\epsilon}{\rightarrow}(\perp, l s)} \\
& \frac{a=r d\left(x, v^{\prime}\right) \quad v^{\prime} \neq u \quad u, v, v^{\prime} \in \text { Val }}{(r \leftarrow \mathbf{C A S}(x, u, v), l s) \stackrel{a}{\longrightarrow}(\perp, l s[r:=\text { false }])} \\
& \frac{a=u p d^{\mathrm{RA}}(x, u, v) \quad u, v \in V a l}{(r \leftarrow \mathbf{C A S}(x, u, v), l s) \stackrel{a}{\rightarrow}(\perp, l s[r:=t r u e])} \quad \frac{a=u p d^{\mathrm{RA}}(x, u, u+1) \quad u \in V a l}{(r \leftarrow \operatorname{FAl}(x), l s) \stackrel{a}{\rightarrow}(\perp, l s[r:=u])} \\
& \frac{(D, l s) \stackrel{a}{\rightarrow}\left(D^{\prime}, l s^{\prime}\right)}{(C[\perp], l s) \stackrel{\epsilon}{\rightarrow}(C, l s)} \quad \frac{a}{(C[D], l s) \stackrel{a}{L}_{L}\left(C\left[D^{\prime}\right], l s^{\prime}\right)} \\
& \mathrm{CLI} \frac{(P(t), \rho(t)) \stackrel{a}{\rightarrow}(C, l s) \quad a \in \operatorname{Act}_{\epsilon}}{(P, \rho) \stackrel{a}{\rightarrow}_{t}(P[t:=C], \rho[t:=l s])} \quad \operatorname{LiB} \frac{(P(t), \rho(t)) \stackrel{a}{\rightarrow}_{L}(C, l s) \quad a \in \text { Act }_{\epsilon}}{(P, \rho) \stackrel{a}{\rightarrow}_{L, t}(P[t:=C], \rho[t:=l s])}
\end{aligned}
$$

Figure 4: Program semantics, where $I F=$ if $B$ then $C_{1}$ else $C_{2}$ and $W H I L E=$ while $B$ do $C$

The operational semantics for this language is defined in three parts. The program semantics fixes the steps that the concurrent program can take. This gives rise to transitions $(P, \rho) \stackrel{a}{\rightarrow}_{t}\left(P^{\prime}, \rho^{\prime}\right)$ of a thread $t$ where $P$ and $P^{\prime}$ are programs, $\rho$ and $\rho^{\prime}$ is the state of local variables and $a$ is an action (possibly the silent action $\epsilon$, see below). The program semantics is combined with a memory semantics which reflects the $\mathrm{C} 11$ state, and in particular the write actions from which a read action can read. Finally, there is the object semantics, which defines the abstract semantics of the object at hand.

We assume that the set of actions is given by Act. We let $\epsilon \notin$ Act be a silent action and let Act $_{\epsilon}=$ Act $\cup\{\epsilon\}$.

In the program semantics, we assume a function $\rho \in T i d \rightarrow($ LVar $\rightarrow$ Val $)$, which returns the local state for the given thread. We assume that the local variables of threads are disjoint, i.e., if $t \neq t^{\prime}$, then $\operatorname{dom}(\rho(t)) \cap \operatorname{dom}\left(\rho\left(t^{\prime}\right)\right)=\emptyset$. For an expression $E$ over local variables, we write $\llbracket E \rrbracket_{l s}$ for the value of $E$ in local state $l s$; we write $l s[r:=v]$ to state that $l s$ remains unchanged except for the value of local variable $r$ which becomes $v$.

We use $C[D]$ to denote the program $C$ with the leftmost innermost hole filled by $D$. If $D=\perp$, we proceed with the execution of $C$, otherwise we execute $D$. Note that if $D$ terminates with a 
value (due to a method call that returns a value), then the hole contains a value and execution may proceed by either using the rule for $r:=v$ or the rule for $v ; C_{2}$, both of which are present in Figure 4. The last two rules, CLI and LIB, lift the transitions of threads to a transition of a client and library program, respectively. These are distinguished by the subscript $L$, which only appears in transitions corresponding to the library.

The rules in Figure 4 allow for all possible values for any read. We constrain these values with respect to a memory semantics (formalised by $\stackrel{a}{\rightarrow} t$ ), which is described for reads, writes and updates in Section 3.3 and for abstract objects in Section 4. The combined semantics brings together a client state $\gamma$ and library state $\beta$ as follows.

$$
\begin{array}{cc}
\frac{(P, \rho) \stackrel{\epsilon}{\rightarrow}_{t}\left(P^{\prime}, \rho^{\prime}\right)}{(P, \rho, \gamma, \beta) \Longrightarrow\left(P^{\prime}, \rho^{\prime}, \gamma, \beta\right)} & \frac{(P, \rho) \stackrel{\epsilon}{\rightarrow}_{L, t}\left(P^{\prime}, \rho^{\prime}\right)}{(P, \rho, \gamma, \beta) \Longrightarrow\left(P^{\prime}, \rho^{\prime}, \gamma, \beta\right)} \\
\frac{(P, \rho) \stackrel{a}{\longrightarrow}_{t}\left(P^{\prime}, \rho^{\prime}\right)}{\gamma, \beta \stackrel{a}{\rightarrow} t \gamma^{\prime}, \beta^{\prime}} & (P, \rho) \stackrel{a}{\longrightarrow}_{L, t}\left(P^{\prime}, \rho^{\prime}\right) \\
(P, \rho, \gamma, \beta) \Longrightarrow\left(P^{\prime}, \rho^{\prime}, \gamma^{\prime}, \beta^{\prime}\right) & \beta, \gamma \stackrel{a}{\rightarrow} \beta^{\prime}, \gamma^{\prime} \\
(P, \rho, \gamma, \beta) \Longrightarrow\left(P^{\prime}, \rho^{\prime}, \gamma^{\prime}, \beta^{\prime}\right)
\end{array}
$$

These rules ensure, for example, that a read only returns a value allowed by the underlying memory model. In Section 4 we introduce additional rules so that the memory model also contains actions corresponding to method calls on an abstract object.

Note that the memory semantics (see Section 3.3 and Section 4) defined by $\gamma, \beta \stackrel{a}{\rightarrow \rightarrow t} \gamma^{\prime}, \beta^{\prime}$ assumes that $\gamma$ is the state of the component being executed and $\beta$ is the state of the context. For a client step, we have that $\gamma$ is the executing component state and $\beta$ is the context state, where as for a library step, these parameters are swapped.

\subsection{Memory Semantics}

Next, we detail the modularised memory semantics, which builds on an earlier monolithic semantics 5, which is a timestamp-based revision of an earlier operational semantics 9. Our present extension is a semantics that copes with client-library interactions in weak memory. Namely, it describes how synchronisation (in our example release-acquire synchronisation) in one component affects thread views in another component. The semantics accommodates both client synchronisation affecting a library, and vice versa.

Component State. We assume Act denotes the set of actions. Following [5], each global write is represented by a pair $(a, q) \in \operatorname{Act} \times \mathbb{Q}$, where $a$ is a write action, and $q$ is a rational number that we use as a timestamp corresponding to modification order (cf. [18, 11, 25]). The set of modifying operations within a component that have occurred so far is recorded in ops $\subseteq$ Act $\times \mathbb{Q}$. Unlike prior works, to accommodate (abstract) method calls of a data structures, we record abstract operations in general, as opposed to writes only.

Each state must record the operations that are observable to each thread. To achieve this, we use two families of functions from global variables to writes (cf. [25, 19]).

- A thread view function tview $t_{t} \in G$ Var $\rightarrow$ ops that returns the viewfront of thread $t$. The thread $t$ can read from any write to variable $x$ whose timestamp is not earlier than $\operatorname{tview}_{t}(x)$. Accordingly, we define, for each state $\gamma$, thread $t$ and global variable $x$, the set of observable writes, where $\operatorname{tst}(w)=q$ denotes $w$ 's timestamp:

$$
\begin{aligned}
\gamma . \operatorname{Obs}(t, x)=\{(a, q) \in \gamma . \mathrm{ops} \mid & \operatorname{var}(a)=x \\
& \left.\wedge \operatorname{tst}\left(\gamma \cdot \operatorname{tview}_{t}(x)\right) \leq q\right\}
\end{aligned}
$$


- A modification view function $\operatorname{mview}_{w} \in G \operatorname{Var} \rightarrow$ Act $\times \mathbb{Q}$ that records the viewfront of write $w$, i.e., the viewfront of the thread that executed $w$ immediately after $w$ 's execution. We use mview $w$ to compute a new value for tview $w_{t}$ if a thread $t$ synchronizes with $w$, i.e., if $w \in \mathbf{W}_{\mathbf{R}}$ and another thread executes an $e \in \mathrm{R}_{\mathrm{A}}$ that reads from $w$.

The client cannot directly access writes in the library, therefore the thread view function must map to writes within the same component. On the other hand, synchronisation in a component can affect thread views in another (as discussed in Section 2), thus the modification view function may map to operations across the system.

Finally, our semantics maintains a set cvd $\subseteq$ ops. In C11 RAR, each update action occurs in modification order immediately after the write that it reads from [9]. This property ensures the atomicity of updates. We disallow any newer modifying operation (write or update) from intervening between any update and the write or update that it reads from. As we explain below, covered writes are those that are immediately prior to an update in modification order, and new write actions never interact with a covered write.

Initialisation. Suppose $\operatorname{GVar}_{C}=\left\{x_{1}, \ldots, x_{n}\right\}, \operatorname{GVar}_{L}=\left\{y_{1}, \ldots, y_{n}\right\}, \operatorname{LVar}=\left\{r_{1}, \ldots, r_{m}\right\}$, $k_{1}, \ldots, k_{n}, l_{1}, \ldots, l_{m} \in \mathrm{Val}$, and Init $=x_{1}:=k_{1} ; \ldots, x_{n}:=k_{n} ;\left[r_{1}:=l_{1} ;\right] \ldots\left[r_{m}:=l_{m} ;\right]$, where we use the notation $\left[r_{i}:=l_{i} ;\right]$ to mean that the assignment $r_{i}:=l_{i}$ may optionally appear in Init. Thus each shared variable is initialised exactly once and each local variable is initialised at most once. The initial values of the state components are then as follows, where we assume 0 is the initial timestamp, $t$ is a thread, $x_{i} \in G \operatorname{Var}_{C}$ and $y_{i} \in G \operatorname{Var}_{L}$

$$
\begin{aligned}
& \gamma_{\text {Init }} . \text { ops }=\left\{\left(w r\left(x_{1}, k_{1}\right), 0\right), \ldots,\left(w r\left(x_{n}, k_{n}\right), 0\right)\right\} \\
& \beta_{\text {Init }} . \text { ops }=\left\{\left(w r\left(y_{1}, k_{1}\right), 0\right), \ldots,\left(w r\left(y_{n}, k_{n}\right), 0\right)\right\} \\
& \gamma_{\text {Init }} \text {.tview } t\left(x_{i}\right)=\left(w r\left(x_{i}, k_{i}\right), 0\right) \\
& \beta_{\text {Init.tview }}\left(y_{i}\right)=\left(w r\left(y_{i}, k_{i}\right), 0\right) \\
& \gamma_{\text {Init }} \text {.mview } x_{i}=\beta_{\text {Init }} \text {.mview } y_{i}=\gamma_{\text {Init }} \text {.tview }{ }_{t} \cup \beta_{\text {Init }} \text {. } \text { tview }_{t} \\
& \gamma_{\text {Init }} \cdot \mathrm{cvd}=\beta_{\text {Init }} \cdot \mathrm{cvd}=\emptyset
\end{aligned}
$$

The local state component of each thread must also be compatible with Init, i.e., for each $t$ if $r_{i} \in \operatorname{dom}(l s t(t))$ we have that $(l s t(t))\left(r_{i}\right)=l_{i}$ provided $r_{i}:=l_{i}$ appears in Init. We let $l_{s} t_{\text {Init }}$ be the local state compatible with Init and let $\Gamma_{\text {Init }}=\left(l s t_{\text {Init }}, \gamma_{\text {Init }}, \beta_{\text {Init }}\right)$.

Transition semantics. The transition relation of our semantics for global reads and writes is given in Figure 5 and builds on an earlier semantics that does not distinguish the state of the context [5]. Each transition $\gamma, \beta \stackrel{a}{\rightarrow} \rightarrow t \gamma^{\prime}, \beta^{\prime}$ is labelled by an action $a$ and thread $t$ and updates the target state $\gamma$ (the state of component being executed) and the context $\beta$.

READ transition by thread $t$. Assume that $a$ is either a relaxed or acquiring read to variable $x, w$ is a write to $x$ that $t$ can observe (i.e., $(w, q) \in \gamma \cdot \operatorname{Obs}(t, x)$ ), and the value read by $a$ is the value written by $w$. Each read causes the viewfront of $t$ to be updated. For an unsynchronised read, tview ${ }_{t}$ is simply updated to include the new write. A synchronised read causes the executing thread's view of the executing component and context to be updated. In particular, for each variable $x$, the new view of $x$ will be the later (in timestamp order) of either $\operatorname{tview}_{t}(x) \operatorname{or}_{\operatorname{mview}}(x)$. To express this, we use an operation that combines two views $V_{1}$ and $V_{2}$, by constructing a new view from $V_{1}$ by taking the later view of each variable:

$$
V_{1} \otimes V_{2}=\lambda x \in \operatorname{dom}\left(V_{1}\right) \text {. if } \operatorname{tst}\left(V_{2}(x)\right) \leq \operatorname{tst}\left(V_{1}(x)\right) \text { then } V_{1}(x) \text { else } V_{2}(x)
$$




$$
\begin{aligned}
& a \in\left\{r d(x, n), r d^{\mathrm{A}}(x, n)\right\} \quad(w, q) \in \gamma \cdot \operatorname{Obs}(t, x) \quad \operatorname{wrval}(w)=n \\
& \text { tview }^{\prime}= \begin{cases}\gamma \cdot \text { tview }_{t} \otimes \gamma \cdot \text { mview }_{(w, q)} & \text { if }(w, a) \in \mathrm{W}_{\mathrm{R}} \times \mathrm{R}_{\mathrm{A}} \\
\gamma \cdot \text { tview }_{t}[x:=(w, q)] & \text { otherwise }\end{cases} \\
& \text { ctview }^{\prime}= \begin{cases}\beta . \text { tview }_{t} \otimes \gamma \cdot \text { mview }_{(w, q)} & \text { if }(w, a) \in \mathrm{W}_{\mathrm{R}} \times \mathrm{R}_{\mathrm{A}} \\
\beta . \text { tview }_{t} & \text { otherwise }\end{cases}
\end{aligned}
$$

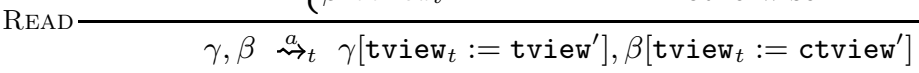

$$
\begin{aligned}
& a \in\left\{w r(x, n), w r^{\mathrm{R}}(x, n)\right\} \quad(w, q) \in \gamma \cdot \mathrm{Obs}(t, x) \backslash \gamma \cdot \mathrm{cvd} \\
& \text { fresh }_{\gamma}\left(q, q^{\prime}\right) \quad \text { ops }^{\prime}=\gamma . \text { ops } \cup\left\{\left(a, q^{\prime}\right)\right\}
\end{aligned}
$$

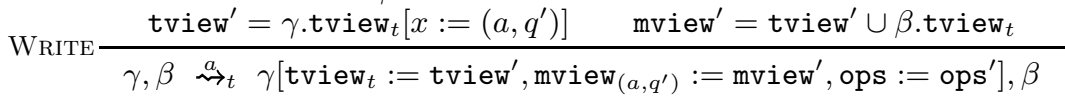

$$
\begin{aligned}
& a=u p d^{\mathrm{RA}}(x, m, n) \quad(w, q) \in \gamma \cdot \mathrm{Obs}(t, x) \backslash \gamma \cdot \operatorname{cvd} \quad \operatorname{wrval}(w)=m \\
& \operatorname{fresh}_{\gamma}\left(q, q^{\prime}\right) \quad \text { ops }^{\prime}=\gamma . \text { ops } \cup\left\{\left(a, q^{\prime}\right)\right\} \\
& \mathrm{cvd}^{\prime}=\gamma \cdot \operatorname{cvd} \cup\{(w, q)\} \quad \text { mview }^{\prime}=\text { tview }^{\prime} \cup \text { ctview }^{\prime} \\
& \text { tview }^{\prime}= \begin{cases}\gamma \cdot \operatorname{tview}_{t}\left[x:=\left(a, q^{\prime}\right)\right] \otimes \gamma \cdot \text { mview }_{(w, q)} & \text { if } w \in \mathrm{W}_{\mathrm{R}} \\
\gamma \cdot \text { tview }_{t}\left[x:=\left(a, q^{\prime}\right)\right] & \text { otherwise }\end{cases} \\
& \text { ctview }^{\prime}= \begin{cases}\beta . \text { tview }_{t} \otimes \gamma \cdot \text { mview }_{(w, q)} & \text { if } w \in \mathrm{W}_{\mathrm{R}} \\
\beta . \text { tview }_{t} & \text { otherwise }\end{cases}
\end{aligned}
$$

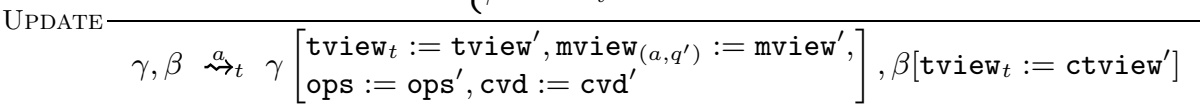

Figure 5: Transition relation for reads, writes and updates of the memory semantics

WRITE transition by thread $t$. A write transition must identify the write $(w, q)$ after which $a$ occurs. This $w$ must be observable and must not be covered - the second condition preserves the atomicity of read-modify-write updates. We must choose a fresh timestamp $q^{\prime} \in \mathbb{Q}$ for $a$, which for a C11 state $\gamma$ is formalised by $\operatorname{fresh}_{\gamma}\left(q, q^{\prime}\right)=q<q^{\prime} \wedge \forall w^{\prime} \in \gamma$.ops. $q<\operatorname{tst}\left(w^{\prime}\right) \Rightarrow q^{\prime}<\operatorname{tst}\left(w^{\prime}\right)$. That is, $q^{\prime}$ is a new timestamp for variable $x$ and that $\left(a, q^{\prime}\right)$ occurs immediately after $(w, q)$. The new write is added to the set ops.

We update $\gamma$.tview $t$ to include the new write, which ensures that $t$ can no longer observe any writes prior to $\left(a, q^{\prime}\right)$. Moreover, we set the viewfront of $\left(a, q^{\prime}\right)$ to be the new viewfront of $t$ in $\gamma$ together with the thread viewfront of the environment state $\beta$. If some other thread synchronises with this new write in some later transition, that thread's view will become at least as recent as t's view at this transition. Since mview keeps track of the executing thread's view of both the component being executed and its context, any synchronisation through this new write will update views across components.

UPDATE transition by thread $t$. These transitions are best understood as a combination of the read and write transitions. As with a write transition, we must choose a valid fresh timestamp $q^{\prime}$, and the state component ops is updated in the same way. State component mview includes information from the new view of the executing thread $t$. As discussed earlier, in UPDATE transitions it is necessary to record that the write that the update interacts with is now covered, which is achieved by adding that write to cvd. Finally, we must compute a new thread view, which is similar to a READ transition, except that the thread's new view always includes the new write 
introduced by the update.

\section{Abstract object semantics}

The rules in Figure 5 provide a semantics for read, write and update operations for component programs within an executing context and can be used to model clients and libraries under RC11 RAR. These rules do not cover the behaviour of abstract objects, which we now consider.

There have been many different proposals for specifying and verifying concurrent objects memory [20, 3, 14, 21, 26, 8, 13, since there are several different objectives that must be addressed. These objectives are delicately balanced in linearizability [17, the most well-used consistency condition for concurrent objects. Namely, linearizability ensures: 1 . The abstract specification is explainable with respect to a sequential specification. 2. Correctness is compositional, i.e., any concrete execution of a system comprising two linearizable objects is itself linearizable. 3. Correctness ensures contextual (aka observational) refinement, i.e., the use of a linearizable implementation within a client program in place of its abstract specification does not induce any new behaviours in the client program.

There is however an inherent cost to linearizability stemming from the fact that the effect of each method call must take place before the method call returns. In the context of weak memory, this restriction induces additional synchronisation that may not necessarily be required for correctness [27. Therefore, over the years, several types of relaxations to the above requirements have been proposed [20, 3, 14, 21, 26, 8, 13.

General data structures present many different design choices at the abstract level [26], but discussing these now detracts from our main contribution, i.e., the integration and verification of clients and libraries in a weak memory model. Therefore, we restrict our attention to an abstract lock object, which is sufficient to highlight the main ideas. Locks have a clear ordering semantics (each new lock acquire and lock release operation must have a larger timestamp than all other existing operations) and synchronisation requirements (there must be a release-acquire synchronisation from the lock release to the lock acquire).

To enable proofs of contextual refinement (see Section 6), we must ensure corresponding method calls return the same value at the abstract and concrete levels. To this end, we introduce a special variable rval to each local state that stores the value that each method call returns.

Example 1 (Abstract lock). Consider the specification of a lock with methods Acquire, and Release. Each method call of the lock is indexed by a subscript to uniquely identify the method call. For the lock, the subscript is a counter indicating how many lock operations have been executed and is used in the example proof in Section 5 .

$$
\begin{gathered}
\operatorname{ACquire} \frac{a=\text { l.acquire }_{n} \quad l s^{\prime}=l s[\text { rval }:=\text { true }]}{(\text { l.Acquire }(), l s) \stackrel{a}{\rightarrow}\left(\text { true }, l s^{\prime}\right)} \\
\text { RELEASE } \frac{a=\text { l.release }_{n} \quad l s^{\prime}=l s[\text { rval }:=\perp]}{(\text { l.Release }(), l s) \stackrel{a}{\rightarrow}\left(\perp, l s^{\prime}\right)}
\end{gathered}
$$

Locks, by default are synchronising. That is, in the memory semantics, a (successful) acquire requires the operation to synchronise with most recent lock release (in a manner consistent with release-acquire semantics), so that any writes that are happens-before ordered before the release are 


$$
\begin{aligned}
& a=\text { l.acquire }_{n} \quad b=\text { l.acquire }_{n}(t) \quad(w, q) \in \gamma . \text { ops } \quad w \in\left\{\text { l.init }_{0}, \text { l.release }_{n-1}\right\} \\
& \text { ops }^{\prime}=\gamma . \text { ops } \cup\left\{\left(b, q^{\prime}\right)\right\} \quad \begin{array}{c}
q=\max T S(l, \gamma) \quad q<q^{\prime} \\
\text { mview }^{\prime}=\text { tview }^{\prime} \cup \text { ctview }^{\prime} \quad \operatorname{cvd}^{\prime}=\sigma . c v d \cup\{(w, q)\}
\end{array}
\end{aligned}
$$

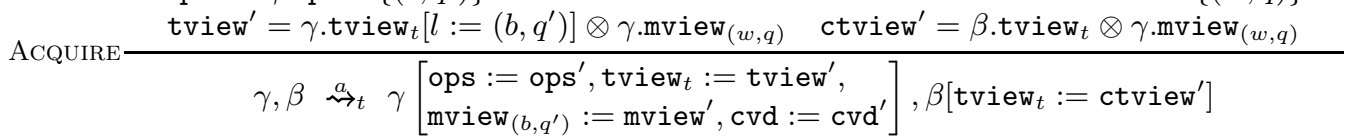

$$
\begin{aligned}
& a=\text { l.release }_{n} \quad w=\text { l. }_{\text {acquire }} \text {. }_{-1}(t) \quad(w, q) \in \gamma . \text { ops } \quad q=\operatorname{maxTS}(l, \gamma) \quad q<q^{\prime} \\
& \operatorname{RELEASE} \frac{\mathrm{ops}^{\prime}=\gamma . \mathrm{ops} \cup\left\{\left(a, q^{\prime}\right)\right\} \quad \text { tview }^{\prime}=\gamma \cdot \text { tview }_{t}\left[x:=\left(a, q^{\prime}\right)\right] \quad \text { mview }^{\prime}=\text { tview }^{\prime} \cup \beta \text {.tview } t}{}
\end{aligned}
$$

Figure 6: Operational semantics for lock acquire and release

visible to the thread that acquires the lock. The initial state of an abstract lock $l, \beta_{\text {Init }}$, is given by:

$$
\begin{aligned}
& \beta_{\text {Init.ops }}=\left\{\left(\text { l.init }_{0}, 0\right)\right\} \quad \gamma_{\text {Init }} . \text { tview }_{t}(l)=\left(\text { l.init }_{0}, 0\right) \\
& \gamma_{\text {Init. }} \cdot \operatorname{cvd}=\emptyset
\end{aligned}
$$

We also obtain the rules below, where we assume $\gamma$ is the state of the lock and $\beta$ is the state of the client.

To record the thread that currently owns the lock, we derive a new action, $b$, from the action a of the program semantics. Action $(w, q)$ represents the method that is observed by the acquire method, which must be an operation in $\gamma$. ops such that $q$ has the maximum timestamp for $l$ (i.e., $q=\max T S(l, \gamma)$. The new timestamp $q^{\prime}$ must be larger than $q$. We create a new component state $\gamma^{\prime}$ from $\gamma$ by

- inserting $\left(b, q^{\prime}\right)$ into $\gamma$. ops;

- updating tview to $\mathrm{tview}^{\prime}$, where tview' synchronises with the previous thread view in $\gamma$ to include information from the modification view of $(w, q)$, and updates $t$ 's view of $l$ to include the new operation $\left(b, q^{\prime}\right)$;

- updating the contextual thread view for t to ctview', where ctview' synchronises with the previous thread view in the context state $\beta$ to include information from the modification view of $(w, q)$; and

- updating the modification view for the new operation $\left(b, q^{\prime}\right)$ to mview', where mview' contains the view of $t$.

Finally, the context state $\beta^{\prime}$ updates the thread view of t to ctview' since synchronisation with a release may cause the view to be updated.

A lock release, simply introduces a new operation with a maximal timestamp, provided that the thread executing the release currently holds the lock.

\section{Client-library verification}

Having formalised the semantics of clients and libraries in a weak memory setting, we now work towards verification of (client) programs that use such libraries. 


\subsection{Assertion language}

In our proof, we use observability assertions, which describe conditions for a thread to observe a specific value for a given variable. Unlike earlier works, our operational semantics covers clients and their libraries, and hence operates over pairs of states.

Possible observation, denoted $\langle x=u\rangle_{t}$, means that thread $t$ may observe value $u$ for $x[5]$. We extend this concept to cope with abstract method calls as follows. In particular, for an object $o$ and method $m$, we use $\langle o . m\rangle_{t}$ to denote that thread $t$ can observe o.m.

$$
\begin{aligned}
\langle x=n\rangle_{t}(\sigma) & \equiv \exists w \in \sigma . \operatorname{Obs}(t, x) . \operatorname{wrval}(w)=n \\
\langle o . m\rangle_{t}(\sigma) & \equiv \exists q .(o . m, q) \in \sigma . \operatorname{ops} \wedge q \geq \sigma . \operatorname{tview}_{t}(o)
\end{aligned}
$$

To distinguish possible observation in clients and libraries, we introduce the following notation, where $\gamma$ and $\beta$ are the client and library states, respectively, and $p$ is either a valuation (i.e., $x=n$ ) or an abstract method call (i.e., o.m):

$$
\langle p\rangle_{t}^{C}(\gamma, \beta) \equiv\langle p\rangle_{t}(\gamma) \quad\langle p\rangle_{t}^{L}(\gamma, \beta) \equiv\langle p\rangle_{t}(\beta)
$$

Definite observation, denoted $[x=u]_{t}$, means that thread $t$ can only see the last write to $x$, and that this write has written value $u$. We define the last write to $x$ in a set of writes $W$ as:

$$
\begin{aligned}
\operatorname{last}(W, x)=w \equiv & w \in\{w \in W \mid \operatorname{var}(w)=x\} \wedge \\
& \left(\forall w^{\prime} \in W_{\mid x} . \operatorname{tst}\left(w^{\prime}\right) \leq \operatorname{tst}(w)\right)
\end{aligned}
$$

We define the definite observation of a view function, view with respect to a set of writes as follows:

$$
\begin{aligned}
& \operatorname{dview}(\operatorname{view}, W, x)=n \\
\equiv & \operatorname{view}(x)=\operatorname{last}(W, x) \wedge \operatorname{wrval}(\operatorname{last}(W, x))=n
\end{aligned}
$$

The first conjunct ensures that the viewfront of view for $x$ is the last write to $x$ in $W$, and the second conjunct ensures that the value written by the last write to $x$ in $W$ is $n$. For a variable $x$, thread $t$ and value $n$, we define:

$$
[x=n]_{t}(\sigma) \equiv \operatorname{dview}\left(\sigma . \text { tview }_{t}, \sigma . \text { ops } \cap \mathrm{W}, x\right)=n
$$

The extension of definite observation assertions to abstract method calls is straightforward to define. Namely we have:

$$
\begin{aligned}
& {[o . m]_{t}(\sigma) \equiv \sigma . \text { tview }_{t}(o)=\max T S(o, \sigma) \wedge} \\
& (o . m, \max T S(o, \sigma)) \in \sigma . o p s
\end{aligned}
$$

As with possible observations, we lift definite observation predicates to state spaces featuring clients and libraries:

$$
[p]_{t}^{C}(\gamma, \beta) \equiv[p]_{t}(\gamma) \quad[p]_{t}^{L}(\gamma, \beta) \equiv[p]_{t}(\beta)
$$

Conditional observation, denoted $\langle x=u\rangle[y=v]_{t}$, means that if thread $t$ synchronises with a write to variable $x$ with value $u$, it must subsequently observe value $v$ for $y$. For variables $x$ and $y$, thread $t$ and values $u$ and $v$, we define:

$$
\begin{aligned}
& \langle x=u\rangle[y=v]_{t}(\sigma) \\
& \equiv \quad \forall w \in \sigma . \operatorname{Dbs}(t, x) . \operatorname{wrval}(w)=u \Rightarrow \\
& \operatorname{act}(w) \in \mathbf{W}_{\mathrm{R}} \wedge \operatorname{dview}\left(\sigma . \operatorname{mview}_{w}, \sigma . o p s, y\right)=v
\end{aligned}
$$


This is a key assertion used in message passing proofs [5, 18] since it guarantees an observation property on a variable, $y$, via a synchronising read of another variable, $x$.

As with possible and definite assertions, conditional assertions can generalised to objects and extended to pairs of states describing a client and its library. However, unlike possible and definition observations assertions, conditional observation enables one to describe view synchronisation across different states. For example, consider the following, which enables conditional observation of an abstract method to establish a definite observation assertion for the thread view of the client. We assume a set Sync $\subseteq$ Act that identifies a set of synchronising abstract actions.

$$
\begin{gathered}
\langle o . m\rangle^{L}[y=v]_{t}^{C}(\gamma, \beta) \\
\equiv \text { o.m } \in \text { Sync } \wedge \forall q .(\text { o.m }, q) \in \sigma . \text { ops } \wedge q \geq \gamma . \text { tview }_{t}(o) \Rightarrow \\
\text { dview }\left(\gamma . \text { mview }_{(o . m, q)}, \beta . o p s, y\right)=v
\end{gathered}
$$

It is possible to define other variations, e.g., conditional observation synchronisation from clients to libraries, but we leave out the details of these since they are straightforward to construct.

Covered operations, denoted $\mathbf{C}_{x}^{u}$, where $x$ is a variable and $u$ a value. Recall from the ACQUIRE rule that a new acquire operation causes the immediately prior (release) operation l.release $_{n-1}$ to be covered so that no later acquire can be inserted between l.release $_{n-1}$ and the new acquire. To reason about this phenomenon over states, we use:

$$
\begin{aligned}
\mathbf{C}_{o . m}(\sigma) \equiv & \forall(w, q) \in \sigma . \mathrm{ops}_{\mid o} \backslash \sigma . \mathrm{cvd} . \\
& w=o . m \wedge q=\operatorname{maxTS}(o, \sigma)
\end{aligned}
$$

where $\sigma . \mathrm{ops}_{\mid o}$ is the set of operations over object $o$.

Hidden value, denoted $\mathbf{H}_{o . m}$, states that the operation $o . m$ exists, but all of these are hidden from interaction. In proofs, such assertions limit the values that can be returned.

$$
\begin{aligned}
\mathbf{H}_{o . m}(\sigma) \equiv \quad & (\exists q .(o . m, q) \in \sigma . \mathrm{ops}) \wedge \\
& (\forall q .(o . m, q) \in \sigma . \mathrm{ops} \Rightarrow(o . m, q) \in \sigma . \mathrm{cvd})
\end{aligned}
$$

Both covered and hidden-value assertions can be lifted to pairs of states and can be used to reason about standard writes, as opposed to method calls (details omitted).

\subsection{Hoare Logic for C11 and Abstract Objects}

Since we have an operational semantics, the assertions in Section 5.1 can be integrated into standard Hoare-style proof calculus in a straightforward manner [5, 6]. The only differences are the state model (which is a weak memory state, as opposed to mappings from variables to values) and the atomic components (which may include reads of global variables, and, in this paper, abstract method calls).

Following [5, 6], we let $\Sigma_{C}$ and $\Sigma_{L}$ to be the set of all possible global state configurations of the client and library, respectively and let $\Sigma_{C 11}=(L V a r \rightarrow \operatorname{Val}) \times \Sigma_{C} \times \Sigma_{L}$ be the set of all possible client-library C11 states. Predicates over $\Sigma_{C 11}$ are therefore of type $\Sigma_{C 11} \rightarrow \mathbb{B}$. This leads to the following definition of a Hoare triple, which we note is the same as the standard definition - the only difference is that the state component is of type $\Sigma_{C 11}$.

Definition 2. Suppose $p, q \in \Sigma_{C 11} \rightarrow \mathbb{B}, P \in$ Prog and $\mathbf{E}=\lambda t:$ Tid. $\perp$. The semantics of a Hoare triple under partial correctness is given by: 


$$
\begin{gathered}
\{p\} \operatorname{Init}\{q\}=q\left(\Gamma_{\text {Init }}\right) \\
\{p\} \operatorname{Init} ; P\{q\}=\exists r .\{p\} \mathbf{I n i t}\{r\} \wedge\{r\} P\{q\} \\
\{p\} P\{q\}=\forall \rho, \gamma, \beta, \rho^{\prime}, \gamma^{\prime}, \beta^{\prime} . p(\rho, \gamma, \beta) \wedge \\
\quad(P, \rho, \gamma, \beta) \Longrightarrow \Longrightarrow^{*}\left(\mathbf{E}, \rho^{\prime}, \gamma^{\prime}, \beta^{\prime}\right) \Rightarrow q\left(\rho^{\prime}, \gamma^{\prime}, \beta^{\prime}\right)
\end{gathered}
$$

This definition (in the context of RC11 [23]) allows all standard Hoare logic rules for compound statements to be reused [5]. Due to concurrency, following Owicki and Gries, one must prove local correctness and interference freedom (or stability) [24, 5, 6, 22. This is also defined in the standard manner. Namely, a statement $R \in A C o m$ with precondition $\operatorname{pre}(R)$ (in the standard proof outline) does not interfere with an assertion $p$ iff $\{p \wedge$ pre $(R)\} R\{p\}$. Proof outlines of concurrent programs are interference free if no statement in one thread interferes with an assertion in another thread.

The only additional properties that one must define are on the interaction between atomic commands and predicates over assertions defined in Section [5.1. A collection of rules for reads, writes and updates have been given in prior work 6, 5. Here, we present rules for method calls of the abstract lock object defined in Example 1

In proofs, it is often necessary to reason about particular versions of the lock (i.e., the lock counter). Therefore, we use 1.Acquire $(v)$ and l.Release $(v)$ to denote the transitions that set the lock version to $v$. Also note that in our example proof, it is clear from context whether an assertion refers to the client or library state, and hence, for clarity, we drop the superscripts $C$ and $L$ as used in Section 5.1

The lemma below has been verified in Isabelle/HOL.

Lemma 3. Each of the following holds, where the statements are decorated with the identifier of the executing thread, assuming $\mathrm{m} \in\{$ Acquire, Release $\}$ and $t \neq t^{\prime}$

$$
\begin{aligned}
& \left\{\mathbf{H}_{l_{\text {.release }}}\right\} \text { 1.Acquire }(v)_{\mathrm{t}}\{v>u+1\} \\
& \left\{\mathbf{H}_{\text {l.release }_{u}}\right\} \text { 1.m }(v)_{\mathrm{t}}\left\{\mathbf{H}_{l_{\text {.release }}}\right\} \\
& \left\{\left[l . \text { release }_{u}\right]_{t}\right\} \text { 1.Acquire }(v)_{\mathrm{t}}\left\{\left[l . \text { acquire }_{u+1}\right]_{t}\right\} \\
& \left\{[x=u]_{t}\right\} 1 . \mathrm{m}(v)_{\mathrm{t}^{\prime}}\left\{[x=u]_{t}\right\} \\
& \left\{\left\langle\text { l.release }_{u}\right\rangle[x=n]_{t}\right\} 1 . \text { 1.Acquire }(v)_{\mathrm{t}}\left\{v=u+1 \Rightarrow[x=n]_{t}\right\} \\
& \left\{\neg\left\langle\text { l.release }_{u}\right\rangle_{t^{\prime}} \wedge[x=v]_{t}\right\} \text { 1.Release }(u)_{\mathrm{t}}\left\{\left\langle\text { release }_{u}\right\rangle[x=v]_{t^{\prime}}\right\}
\end{aligned}
$$

\subsection{Example Client-Library Verification}

To demonstrate use of our logic in verification, consider the simple program in Figure 7 which comprises a lock object $l$ and shared client variables $d_{1}$ and $d_{2}$ (both initially 0 ). Thread 1 writes 5 to both $d_{1}$ and $d_{2}$ after acquiring the lock while thread 2 reads $d_{1}$ and $d_{2}$ (into local registers $r_{1}$ and $r_{2}$ ) also after acquiring the lock.

Under SC, it is a standard exercise to show that the program terminates with $r_{1}=r_{2}$ and $r_{i}=0$ or $r_{i}=5$. We show that the lock specification in Section 4 together with the assertion language from Section 5.1 and Owicki-Gries logic from Section 5.2 is sufficient to prove this property. In particular, the specification guarantees adequate synchronisation so that if the Thread 2's lock acquire sees the lock release in Thread 1, it also sees the writes to $d_{1}$ and $d_{2}$. The proof relies on two distinct types of properties: 


\begin{tabular}{|c|c|c|}
\hline \multicolumn{3}{|c|}{$\begin{array}{c}\text { Init: } \quad d_{1}:=0 ; d_{2}:=0 ; \text { l.init }() \\
\left\{\text { Inv } \wedge\left[d_{1}=0\right]_{1} \wedge\left[d_{2}=0\right]_{1} \wedge\left[d_{1}=0\right]_{2} \wedge\left[d_{2}=0\right]_{2}\right\}\end{array}$} \\
\hline Thread 1 & Thread 2 & \\
\hline $1:\left\{\operatorname{Inv} \wedge \mathbf{P}_{1}\right\}$ if 1. Acquire () & $1:\left\{\operatorname{Inv} \wedge \mathbf{Q}_{\mathbf{1}}\right\}$ & 1.Acquire $(r l)$ \\
\hline $2:\left\{I n v \wedge \mathbf{P}_{2}\right\} \quad d_{1}:=5$ & $2:\left\{\operatorname{Inv} \wedge \mathbf{Q}_{\mathbf{2}}\right\}$ & $r_{1} \leftarrow d_{1}$ \\
\hline $3:\left\{\operatorname{Inv} \wedge \mathbf{P}_{\mathbf{3}}\right\}$ & $3:\left\{\operatorname{Inv} \wedge \mathbf{Q}_{\mathbf{3}}\right\}$ & $r_{2} \leftarrow d_{2}$ \\
\hline $4:\left\{\operatorname{Inv} \wedge \mathbf{P}_{4}\right\}$ & $4:\left\{\operatorname{Inv} \wedge \mathbf{Q}_{4}\right\}$ & 1.Release() \\
\hline \multicolumn{3}{|c|}{$5:\left\{\left(r_{1}=0 \wedge r_{2}=0\right) \vee\left(r_{1}=5 \wedge r_{2}=5\right)\right\}$} \\
\hline
\end{tabular}

where assuming $P_{p o}=\left(p c_{2}=1 \Rightarrow \neg\langle\text { l.release }\rangle_{2}\right) \wedge \mathbf{H}_{\text {l.init }_{0}}$, we have

$$
\begin{aligned}
& P_{1}=\left[d_{1}=0\right]_{1} \wedge\left[d_{2}=0\right]_{1} \wedge \\
& Q_{1}^{\prime}=p c_{1}=5 \wedge\left\langle\text { l.release }_{2}\right\rangle\left[d_{1}=5\right]_{2} \wedge\left\langle\text { l.release }_{2}\right\rangle\left[d_{2}=5\right]_{2} \\
& \left(p c_{2}=1 \Rightarrow\left[\text { l.init }_{0}\right]_{1} \wedge\left[\text { l.init }_{0}\right]_{2}\right) \quad Q_{1}=\left(p c_{1} \notin\{2,3,4\} \Rightarrow\left(\left[\text { l.init }_{0}\right]_{2} \wedge\left[d_{1}=0\right]_{2} \wedge\left[d_{2}=0\right]_{2}\right) \vee Q_{1}^{\prime}\right) \\
& \wedge\left(p c_{2} \in\{2,3,4\} \Rightarrow \mathbf{C}_{\text {l. acquire }_{1}}\right) \quad \wedge\left(p c_{1}=1 \Rightarrow\left[\text { l.init }_{0}\right]_{1}\right) \wedge\left(p c_{1}=5 \Rightarrow \mathbf{H}_{\text {l.init }_{0}}\right) \\
& P_{2}=\left[d_{1}=0\right]_{1} \wedge\left[d_{2}=0\right]_{1} \wedge P_{p o} \\
& P_{3}=\left[d_{1}=5\right]_{1} \wedge\left[d_{2}=0\right]_{1} \wedge P_{p o} \\
& P_{4}=\left[d_{1}=5\right]_{1} \wedge\left[d_{2}=5\right]_{1} \wedge P_{p o} \\
& Q_{2}=\left(r l=1 \Rightarrow\left[d_{1}=0\right]_{2} \wedge\left[d_{2}=0\right]_{2}\right) \\
& \wedge\left(r l=3 \Rightarrow\left[d_{1}=5\right]_{2} \wedge\left[d_{2}=5\right]_{2}\right) \\
& Q_{3}=\left(r l=1 \Rightarrow r_{1}=0 \wedge\left[d_{2}=0\right]_{2}\right) \\
& \wedge\left(r l=3 \Rightarrow r_{1}=5 \wedge\left[d_{2}=5\right]_{2}\right) \\
& Q_{4}=\left(r l=1 \Rightarrow r_{1}=0 \wedge r_{2}=0\right) \wedge\left(r l=3 \Rightarrow r_{1}=5 \wedge r_{2}=5\right)
\end{aligned}
$$

Figure 7: Proof outline for lock-synchronisation

- Mutual exclusion: As in SC, no two threads should execute their critical sections at the same time.

- Write visibility: If thread 1 enters its critical section first, its writes to both $d_{1}$ and $d_{2}$ must be visible to thread 2 after thread 2 acquires the lock. Note that this property is not necessarily guaranteed in a weak memory setting since all accesses to $d_{1}$ and $d_{2}$ in Figure 7 are relaxed.

Our proof is supported by the following global invariant:

$$
\text { Inv } \equiv \neg\left(p c_{1} \in\{2,3,4\} \wedge p c_{2} \in\{2,3,4\}\right) \wedge(r l \in\{1,3\})
$$

The first conjunct establishes mutual exclusion, while the second ensures that the lock version written by the acquire in thread 2 is either 1 or 3 , depending on which thread enters its critical section first.

The main purpose of the definite and possible observation assertions is to establish $Q_{1}^{\prime}$ (which appers in $Q_{1}$ ) using rule (6). This predicate helps establish $\left[d_{1}=5\right]_{2}$ and $\left[d_{2}=5\right]_{2}$ in thread 2 whenever thread 2 acquires the lock after thread 1.

The most critical of these assertions is $Q_{1}$, which states that if thread 1 is not executing it's critical section then we either have

- $[\text { l.init }]_{2} \wedge\left[d_{1}=0\right]_{2} \wedge\left[d_{2}=0\right]_{2}$, i.e., thread 2 can definitely see the lock initialisation and definitely observes both $d_{1}$ and $d_{2}$ to have value 0 , or

- $Q_{1}^{\prime}$ holds, i.e., thread 1 has released the lock and has established a state whereby if thread 2 acquires the lock, it will be able to establish the definite value assertions $\left[d_{1}=5\right]_{2}$ and $\left[d_{2}=5\right]_{2}$.

Note that $Q_{1}$ also includes a conjunct $p c_{1}=5 \Rightarrow \mathbf{H}_{\text {l.init }}$, which ensures that if thread 2 enters its critical section after thread 1 has terminated, then it does so because it sees l.release $_{2}$ (as opposed to l.init $_{0}$ ). This means that we can establish $r l=1 \Rightarrow\left[d_{1}=0\right]_{2} \wedge\left[d_{2}=0\right]_{2}$ (i.e., thread 2 has 
acquired the lock first) and $r l=3 \Rightarrow\left[d_{1}=5\right]_{2} \wedge\left[d_{2}=5\right]_{2}$ (i.e., thread 2 has acquired the lock second) in $Q_{2}$. Using these definite value assertions, we can easily establish that the particular values that are loaded into registers $r_{1}$ and $r_{2}$. The lemma has been verified in Isabelle/HOL.

Lemma 4. The proof outline in Figure 7 is valid.

\section{Contextual Refinement}

We now describe what it means to implement a specification so that any client properties that was preserved by the specification is not invalidated by the implementation. We define and prove contextual refinement directly, i.e., without appealing to external correctness conditions over libraries, c.f. linearizability [17, 8, 16, 13, 15].

\subsection{Refinement and Simulation for Weak Memory}

Since we have an operational semantics with an interleaving semantics over weak memory states, the development of our refinement theory closely follows the standard approach under SC 7 .

Suppose $P$ is a program with initialisation Init. An execution of $P$ is defined by a possibly infinite sequence $\Pi_{0} \Pi_{1} \Pi_{2} \ldots$ such that

1. each $\Pi_{i}$ is a 4 -tuple $\left(P_{i}, l s_{i}, \gamma_{i}, \beta_{i}\right)$ comprising a program, local state, global client state and global library state, and

2. $\left(l s_{0}, \gamma_{0}, \sigma_{0}\right)=\left(l s_{\text {Init }}, \gamma_{\text {Init }}, \sigma_{\text {Init }}\right)$, and

3. for each $i$, we have $\Pi_{i} \Longrightarrow \Pi_{i+1}$ as defined in Section 3.2 .

A client trace corresponding to an execution $\Pi_{0} \Pi_{1} \Pi_{2} \ldots$ is a sequence $c t \in \Sigma_{C}^{*}$ such that $c t_{i}=$ $\left(\pi_{2}\left(\Pi_{i}\right)_{\mid C}, \pi_{3}\left(\Pi_{i}\right)\right)$, where $\pi_{n}$ is a projection function that extracts the $n$th component of a given tuple and $l s_{\mid C}$ restricts the given local state $l s$ to the variables in $L \operatorname{Var}_{C}$. Thus each $c t_{i}$ is the global client state component of $\Pi_{i}$.

After a projection, the concrete implementation may contain (finite or infinite) stuttering [7, i.e., consecutive states in which the client state is unchanged. We let rem_stut(ct) be the function that removes all stuttering from the trace $c t$, i.e., each consecutively repeating state is replaced by a single instance of that state. We let $\operatorname{Tr}_{S F}(P)$ denote the set of stutter-free traces of a program $P$, i.e., the stutter-free traces generated from the set of all executions of $P$.

Below we refer to the client that uses the abstract object as the abstract client and the client that uses the object's implementation as the concrete client. The notion of contextual refinement that we develop ensures that a client is not able to distinguish the use of a concrete implementation in place of an abstract specification. In other words, each thread of the concrete client should only be able to observe the writes (and updates) in the client state (i.e., $\gamma$ component) that the thread could already observe in a corresponding of the client state of the abstract client.

First we define trace refinement for weak memory states.

Definition 5 (State and Trace Refinement). We say a concrete state $\gamma_{C}$ is a refinement of an abstract state $\gamma_{A}$, denoted $\left(l s_{A}, \gamma_{A}\right) \sqsubseteq\left(l s_{C}, \gamma_{C}\right)$ iff $l s_{A}=l s_{C}, \gamma_{A} . \mathrm{cvd}=\gamma_{C}$.cvd and for all threads $t$ and $x \in G$ Var, we have $\gamma_{C} \cdot \operatorname{Obs}(t, x) \subseteq \gamma_{A} .0 \mathrm{bs}(t, x)$. We say a concrete trace ct is a refinement of an abstract trace at, denoted at $\sqsubseteq c t$, iff $c t_{i} \sqsubseteq a t_{i}$ for all $i$. 
This now leads to a natural definition of contextual refinement that is based on the refinement of traces.

Definition 6 (Program Refinement). A concrete program $P_{C}$ is a refinement of an abstract program $P_{A}$, denoted $P_{A} \sqsubseteq P_{C}$, iff for any (stutter-free) trace ct $\in \operatorname{Tr}_{S F}\left(P_{C}\right)$ there exists a (stutter-free) trace at $\in \operatorname{Tr}_{S F}\left(P_{A}\right)$ such that at $\sqsubseteq$ ct.

Finally, we obtain a notion of contextual refinement for abstract objects. Suppose $P$ is a program with holes. We let $P[O]$ be the program in which the holes are filled with the operations from object $O$. Note that $O$ may be an abstract object, in which case execution of each method call follows the abstract object semantics (Section 4), or a concrete implementation, in which case execution of each method call follows the semantics of reads, writes and updates (Section 3.2).

Definition 7 (Contextual refinement). We say a concrete object $C O$ is a contextual refinement of an abstract object $A O$ iff for any client program $C$, we have $C[A O] \sqsubseteq C[C O]$.

To verify contextual refinement, we use a notion of simulation, which once again is a standard technique from the literature. The difference in a weak memory setting is the fact that the refinement rules must relate more complex configurations, i.e., tuples of the form $(P, l s t, \gamma, \alpha)$.

The simulation relation, $R$, relates triples $\left(a l s, \gamma_{A}, \alpha\right)$, comprising an abstract local state als, client state $\gamma_{A}$ and library state $\alpha$, with triples $\left(c l s, \gamma_{C}, \beta\right)$ comprising a concrete local state $c l s$, a client state $\gamma_{C}$ and concrete library state $\beta$. The simulation condition must ultimately ensure $\left(a l s_{\mid C}, \gamma_{A}\right) \sqsubseteq\left(c l s_{\mid C}, \gamma_{C}\right)$ at each step as defined in Definition 5. However, since client synchronisation can affect the library state, a generic forward simulation rule is non-trivial to define since it requires one to describe how clients steps affect the simulation relation. We therefore present a simpler use case for libraries that are used by clients that do not perform any synchronisation outside the library itself (e.g., the client in Figure (7). If $\Pi=(P, l s t, \gamma, \alpha)$, we let state $(\Pi)=(l s t, \gamma, \alpha)$ be the state corresponding to $\Pi$.

Definition 8 (Forward simulation for synchronisation-free clients). For an abstract object $A O$ and a concrete object $C O$ and a client $C$ that only synchronises through $A O$ (and $C O$ ), $C[A O] \sqsubseteq C[C O]$ holds if there exists a relation $R$ such that

1. $R\left(\left(a l s, \gamma_{A}, \alpha\right),\left(c l s, \gamma_{C}, \beta\right)\right) \Rightarrow$

als $_{\mid C}=\operatorname{cls}_{\mid C} \wedge \gamma_{A} \cdot \mathrm{cvd}=\gamma_{C} \cdot \mathrm{cvd} \wedge$

$\forall t, x . \gamma_{C} \cdot \operatorname{Obs}(t, x) \subseteq \gamma_{A} \cdot \operatorname{Obs}(t, x) \wedge$ $a l s(t)($ rval $)=\operatorname{cls}(t)($ rval $)$

(client observation)

2. $R\left(\operatorname{state}\left(\Omega_{\text {Init }}\right)\right.$, state $\left.\left(\Pi_{\text {Init }}\right)\right)$ (initialisation)

3. For any concrete configurations $\Pi, \Pi^{\prime}$ and abstract configuration $\Omega$, if $\Pi \Longrightarrow \Pi^{\prime}$ via a step corresponding to $C O$, and $R($ state $(\Omega)$, state $(\Pi))$, then either

- $R(\operatorname{state}(\Omega)$, state $(\Pi))$, or (stuttering step)

- there exists an abstract configuration $\Omega^{\prime}$ such that $\Omega \Longrightarrow \Omega^{\prime}$ and $R\left(\operatorname{state}\left(\Omega^{\prime}\right)\right.$, state $\left.\left(\Pi^{\prime}\right)\right)$. (non-stuttering step)

Theorem 8.1. If $R$ is a forward simulation between $A O$ and $C O$, then for any client that only synchronises through $A O$ (and $C O$ ) we have $C[A O] \sqsubseteq C[C O]$. 


\subsection{Sequence Lock}

The first refinement example is a sequence lock which operates over a single shared variable $(g l b)$.

Init: $g l b=0$

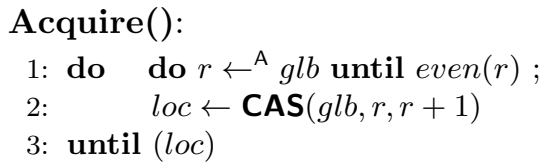

Release():

1: $g l b:={ }^{\mathrm{R}} r+2$

The Acquire operation returns true if, and only if, the CAS on line 2 is successful. Therefore, in order to prove the refinement, we will need to prove that whenever the CAS operation is successful, the abstract object can also successfully acquire the lock maintaining the simulation relation. Also, the read on line 1 and the unsuccessful CAS are stuttering steps and we need to show that when those steps are taken the abstract state remains unchanged and the new concrete state preserves the simulation relation. The Release operation contains only one releasing write on variable $g l b$, which is considered to be a refining step. It is straightforward to show that this operation refines the abstract object release operation. The following proposition has been verified in Isabelle/HOL.

Proposition 9. For synchronisation-free clients, there exists a forward simulation between the abstract lock object and the sequence lock.

\subsection{Ticket Lock}

Our second refinement example is the ticket lock:

Init: $n t=0, s n=0$

\section{Acquire():}

1: $m_{-} t \leftarrow \mathbf{F A l}(n t)$

2: do $s \_n \leftarrow^{\mathrm{A}}$ sn until $m_{-} t=s_{-} n$

Release():

1: $s n: v={ }^{\mathrm{R}} s \_n+1$

The ticket lock has two shared variables $n t$ (next ticket) and $s n$ (serving now). Invocation of Acquire loads the next available ticket into a local register $\left(m_{-} t\right)$ and increases the value of $n t$ by one using a fetch-and-increment (FAI) operation. It then enters a busy loop and reads $s n$ until it sees its own ticket value in $s n$ before it can enter its critical section.

If the read on line 2 of the Acquire operation reads from a write whose value is equal to the value of $m_{-} t$, then the lock is acquired. Therefore we will need to show that if this situation arises, the abstract lock object can also take a step and successfully acquire the lock. We consider the FAI operation on line 1 and the read on line 2 if it reads a value that is not equal to $m_{-} t$ to be a stuttering step. We prove that each of the stuttering and non-stuttering steps preserves the simulation relation. Similar to the previous example, the Release operation consists of only one releasing write to variable $s n$ and it is straightforward to show that this operation refines the abstract release operation. This proof has been mechanised in Isabelle/HOL.

Proposition 10. For synchronisation-free clients, there exists a forward simulation between the abstract lock object and the ticket lock. 


\section{Conclusions}

In this paper, we present a new approach to specifying and verifying abstract objects over weak memory by extending an existing operational semantics for RC11 RAR (which is a fragment of the C11 memory model). We show that our methodology supports two types of verification: (1) proofs of correctness of client programs that use abstract libraries and (2) refinement proofs between abstract libraries and their implementations. Moreover, the operational semantics allows one to execute programs in thread order and accommodates weak memory behaviours via a special encoding of the state. To exploit this operational semantics, we develop an assertion language that describes a thread's observations of client-library states, which is in turn used to verify program invariants and proofs of refinement. The operational semantics, proof rules and example verifications have been mechanised in Isabelle/HOL.

There are now several different approaches to program verification that support different aspects of weak memory using pen-and-paper proofs (e.g., [22, 31, 2, 10]), model checking (e.g., 20, 1]), specialised tools (e.g., [30, 21, 29, 28]), and generalist theorem provers (e.g., [5]). These cover a variety of (fragments of) memory models and proceed via exhaustive checking, specialist separation logics, or Hoare-style calculi.

The idea that abstract methods should specify synchronisation guarantees has been established in earlier work [8, 13], where it has been shown to be necessary for contextual refinement [13] and compositionality [8]. Raad et al 26] have tackled the problem of client-library programs and also consider the C11 memory model.

Krishna et al 21 have developed an approach to verifying implementations of weakly consistent libraries [14. They account for weak memory relaxations by transitioning over a generic happens-before relation encoded within a transition system. On the one hand, this means that their techniques apply to any memory model, but on the other hand, such a happens-before relation must ultimately be supplied.

In future work, it would be interesting to further investigate implementations of other concurrent data types and transactional memory within this operational framework.

\section{References}

[1] P. A. Abdulla, J. Arora, M. F. Atig, and S. N. Krishna. Verification of programs under the release-acquire semantics. In PLDI, pages 1117-1132, 2019.

[2] J. Alglave and P. Cousot. Ogre and Pythia: an invariance proof method for weak consistency models. In G. Castagna and A. D. Gordon, editors, POPL, pages 3-18. ACM, 2017.

[3] M. Batty, M. Dodds, and A. Gotsman. Library abstraction for $\mathrm{C} / \mathrm{C}++$ concurrency. In R. Giacobazzi and R. Cousot, editors, POPL, pages 235-248. ACM, 2013.

[4] M. Batty, S. Owens, S. Sarkar, P. Sewell, and T. Weber. Mathematizing C++ concurrency. In T. Ball and M. Sagiv, editors, POPL, pages 55-66. ACM, 2011.

[5] S. Dalvandi, S. Doherty, B. Dongol, and H. Wehrheim. Owicki-gries reasoning for C11 RAR. In R. Hirschfeld and T. Pape, editors, ECOOP, volume 166 of LIPIcs, pages 11:1-11:26. Schloss Dagstuhl - Leibniz-Zentrum für Informatik, 2020. 
[6] S. Dalvandi, B. Dongol, and S. Doherty. Integrating Owicki-Gries for C11-style memory models into Isabelle/HOL. CoRR, abs/2004.02983, 2020.

[7] W. P. de Roever and K. Engelhardt. Data Refinement: Model-oriented Proof Theories and their Comparison, volume 46 of Cambridge Tracts in Theoretical Computer Science. Cambridge University Press, 1998.

[8] S. Doherty, B. Dongol, H. Wehrheim, and J. Derrick. Making linearizability compositional for partially ordered executions. In C. A. Furia and K. Winter, editors, $i F M$, volume 11023 of LNCS, pages 110-129. Springer, 2018.

[9] S. Doherty, B. Dongol, H. Wehrheim, and J. Derrick. Verifying C11 programs operationally. In Jeffrey K. Hollingsworth and Idit Keidar, editors, PPoPP, pages 355-365. ACM, 2019.

[10] M. Doko and V. Vafeiadis. Tackling real-life relaxed concurrency with fsl++. In ESOP, pages 448-475. Springer, 2017.

[11] S. Dolan, KC Sivaramakrishnan, and A. Madhavapeddy. Bounding data races in space and time. In PLDI, PLDI 2018, pages 242-255, New York, NY, USA, 2018. ACM.

[12] B. Dongol and L. Groves. Contextual trace refinement for concurrent objects: Safety and progress. In ICFEM, volume 10009 of $L N C S$, pages 261-278, 2016.

[13] B. Dongol, R. Jagadeesan, J. Riely, and A. Armstrong. On abstraction and compositionality for weak-memory linearisability. In VMCAI, volume 10747 of LNCS, pages 183-204. Springer, 2018.

[14] M. Emmi and C. Enea. Weak-consistency specification via visibility relaxation. Proc. ACM Program. Lang., 3(POPL):60:1-60:28, 2019.

[15] I. Filipovic, P. W. O'Hearn, N. Rinetzky, and H. Yang. Abstraction for concurrent objects. Theor. Comput. Sci., 411(51-52):4379-4398, 2010.

[16] A. Gotsman and H. Yang. Liveness-preserving atomicity abstraction. In ICALP (2), volume 6756 of $L N C S$, pages 453-465. Springer, 2011.

[17] M. Herlihy and J. M. Wing. Linearizability: A correctness condition for concurrent objects. ACM TOPLAS, 12(3):463-492, 1990.

[18] J.-O. Kaiser, H.-H. Dang, D. D., O. Lahav, and V. Vafeiadis. Strong logic for weak memory: Reasoning about release-acquire consistency in Iris. In Peter Müller, editor, ECOOP, volume 74 of LIPIcs, pages 17:1-17:29. Schloss Dagstuhl - Leibniz-Zentrum fuer Informatik, 2017.

[19] J. Kang, C.-K. Hur, O. Lahav, V. Vafeiadis, and D. Dreyer. A promising semantics for relaxedmemory concurrency. In POPL, pages 175-189. ACM, 2017.

[20] M. Kokologiannakis, A. Raad, and V. Vafeiadis. Model checking for weakly consistent libraries. In PLDI, pages 96-110, 2019.

[21] S. Krishna, M. Emmi, C. Enea, and D. Jovanovic. Verifying visibility-based weak consistency. In P. Müller, editor, ESOP, volume 12075 of Lecture Notes in Computer Science, pages 280307. Springer, 2020. 
[22] O. Lahav and V. Vafeiadis. Owicki-Gries reasoning for weak memory models. In M. M. Halldórsson, K. Iwama, N. Kobayashi, and B. Speckmann, editors, ICALP, volume 9135 of LNCS, pages 311-323. Springer, 2015.

[23] O. Lahav, V. Vafeiadis, J. Kang, C.-K. Hur, and D. Dreyer. Repairing sequential consistency in $\mathrm{C} / \mathrm{C}++11$. In $P L D I$, pages $618-632$. ACM, 2017.

[24] S. S. Owicki and D. Gries. An axiomatic proof technique for parallel programs I. Acta Inf., 6:319-340, 1976.

[25] A. Podkopaev, I. Sergey, and A. Nanevski. Operational aspects of C/C++ concurrency. CoRR, abs/1606.01400, 2016.

[26] A. Raad, M. Doko, L. Rozic, O. Lahav, and V. Vafeiadis. On library correctness under weak memory consistency: specifying and verifying concurrent libraries under declarative consistency models. Proc. ACM Program. Lang., 3(POPL):68:1-68:31, 2019.

[27] P. Sewell, S. Sarkar, S. Owens, F. Z. Nardelli, and M. O. Myreen. x86-tso: a rigorous and usable programmer's model for x86 multiprocessors. Commun. ACM, 53(7):89-97, 2010.

[28] A. J. Summers and P. Müller. Automating deductive verification for weak-memory programs. In D. Beyer and M. Huisman, editors, TACAS, volume 10805 of $L N C S$, pages 190-209. Springer, 2018.

[29] K. Svendsen, J. Pichon-Pharabod, M. Doko, O. Lahav, and V. Vafeiadis. A separation logic for a promising semantics. In A. Ahmed, editor, ESOP, volume 10801 of LNCS, pages 357-384. Springer, 2018.

[30] J. Tassarotti, D. Dreyer, and V. Vafeiadis. Verifying read-copy-update in a logic for weak memory. In D. Grove and S. Blackburn, editors, PLDI, pages 110-120. ACM, 2015.

[31] A. Turon, V. Vafeiadis, and D. Dreyer. GPS: navigating weak memory with ghosts, protocols, and separation. In A. P. Black and T. D. Millstein, editors, OOPSLA, pages 691-707. ACM, 2014. 